\title{
Absence of ghrelin protects against early-onset obesity
}

\author{
Katherine E. Wortley, ${ }^{1}$ Juan-Pablo del Rincon, ${ }^{2}$ Jane D. Murray, ${ }^{1}$ Karen Garcia, ${ }^{1}$ Keiji lida, ${ }^{2}$ \\ Michael O. Thorner, ${ }^{2}$ and Mark W. Sleeman'
}

${ }^{1}$ Regeneron Pharmaceuticals Inc., Tarrytown, New York, USA. 2Department of Medicine, University of Virginia, Charlottesville, Virginia, USA.

\begin{abstract}
The gut peptide ghrelin, the endogenous ligand for the growth hormone secretagogue receptor, has been implicated not only in the regulation of pituitary growth hormone $(\mathrm{GH})$ secretion but in a number of endocrine and nonendocrine functions, including appetitive behavior and carbohydrate substrate utilization. Nevertheless, recent genetic studies have failed to show any significant defects in GH levels, food intake, or body weight in adult ghrelin-deficient ( $\left.\mathrm{Ghrl}^{-/-}\right)$mice. Here we demonstrate that male $\mathrm{Gbrl}^{-/-}$mice are protected from the rapid weight gain induced by early exposure to a high-fat diet 3 weeks after weaning ( 6 weeks of age). This reduced weight gain was associated with decreased adiposity and increased energy expenditure and locomotor activity as the animals aged. Despite the absence of ghrelin, these $\mathrm{Gbrl}^{-/-}$mice showed a paradoxical preservation of the GH/IGF-1 axis, similar to that reported in lean compared with obese humans. These findings suggest an important role for endogenous ghrelin in the metabolic adaptation to nutrient availability.
\end{abstract}

\section{Introduction}

Ghrelin is a 28-aa peptide produced predominantly by the stomach and is the endogenous ligand for the growth hormone secretagogue receptor (GHSR) (1). In addition to potently stimulating growth hormone $(\mathrm{GH})$ secretion from the pituitary $(1)$, ghrelin administration stimulates food intake $(2,3)$ and carbohydrate utilization (2) and increases adiposity in rodents (2), suggesting a role for this hormone in energy balance. Consistent with this idea, ghrelin levels are modulated by changes in nutritional status, such as food deprivation (2) or exposure to a high-fat diet (HFD) $(4,5)$. Further, GHSR is colocalized with neuropeptide Y/agouti-related protein (NPY/AgRP) neurons in the arcuate nucleus of the hypothalamus (6), an area that is responsive to circulating nutrients and hormones related to energy balance (7). The finding that ghrelin stimulates the spontaneous activity of these neurons (8) and increases NPY and AgRP gene expression (9) indicates that this hormone may mediate its effects on food intake and metabolic parameters via interactions with these peptide systems.

However, a recent study of ghrelin-deficient mice by Sun et al. (10) failed to identify any disturbances in growth rate, food intake, or body composition in these mice on either a standard or HFD and found no difference in their behavioral response to a period of food deprivation. A further study by this group also found normal growth rate, food intake, and body composition in Ghsr-deficient mice (11). Thus, these genetic studies question the role of endogenous ghrelin in regulating energy balance and the suitability of the ghrelin pathway as a target for potential antiobesity therapies.

In agreement with Sun et al. (10), we previously reported that ghrelin-deficient $\left(\mathrm{Ghrl}^{-/-}\right)$mice exhibit normal spontaneous food intake patterns and normal basal levels of hypothalamic orexigenic and anorexigenic neuropeptides, with no impairment in

Nonstandard abbreviations used: AgRP, agouti-related protein; ALT, alanine aminotransferase; AST, aspartate aminotransferase; GH, growth hormone; GHR, GH receptor; GHSR, growth hormone secretagogue receptor; HFD, high-fat diet; NPY, neuropeptide Y; RQ, respiratory quotient.

Conflict of interest: The authors have declared that no conflict of interest exists.

Citation for this article: J. Clin. Invest. 115:3573-3578 (2005).

doi:10.1172/JCI26003. feeding (12), indicating that endogenous ghrelin has, at most, a redundant role in the regulation of appetite. However, further analyses of adult $\mathrm{Ghrl}^{-/-}$mice on a HFD demonstrated that endogenous ghrelin plays a prominent role in determining the type of metabolic substrate used to maintain energy balance (i.e., fat vs. carbohydrate). Although a trend toward a lean phenotype on the HFD was apparent in these male mice, no statistically significant body weight differential could be shown (12).

In this study, we subjected $\mathrm{Ghrl}^{-/-}$mice to a HFD 3 weeks after weaning in an attempt to accentuate the phenotype of these mice. The results of these studies show that male $\mathrm{Ghrl}^{-/-}$mice exposed to a HFD at an early age maintain a lean phenotype in association with increased energy expenditure and locomotor activity, suggesting that ghrelin plays an important role in regulating energy balance in young mice.

\section{Results}

Reduced body weight gain and adiposity of male Ghrl-1- mice fed a HFD. To study the effects of ghrelin deficiency on growth, development, and metabolic efficiency, $\mathrm{Ghrl}^{+/+}$and $\mathrm{Ghrl}^{-/-}$mice were placed on a HFD 3 weeks after weaning (at approximately 6 weeks of age). Male Ghrl-/- mice showed no difference in body weight gain compared with littermate control mice $\left(\mathrm{Ghrl}^{+/+}\right)$when maintained on a standard chow diet (Figure 1A). In contrast, male $\mathrm{Ghrl}^{-/-}$mice that were switched to the HFD ( $45 \%$ fat) exhibited a markedly reduced rate of body weight gain over time. This resulted in an approximately 30\% lower body weight in male $\mathrm{Ghrl}^{-/}$mice at 24 weeks of age (Figure 1, A and B), which is similar to the weight attained by the mice on the standard diet. In contrast to male mice, female Ghrl $^{-1-}$ mice maintained on the HFD 3 weeks after weaning did not demonstrate a difference in body weight gain compared with littermate control mice (data not shown).

The consumption of a HFD fed ad libitum changes body composition and metabolic status and alters levels of cholesterol, triglycerides, and insulin in normal C57BL6/J mice. Analysis of body composition at 20 weeks of age showed that lean and fat mass of male $\mathrm{Ghrl}^{+/+}$and $\mathrm{Ghrl}^{-/-}$mice were equivalent when maintained on the standard diet (Figure 1C). In the mice maintained 

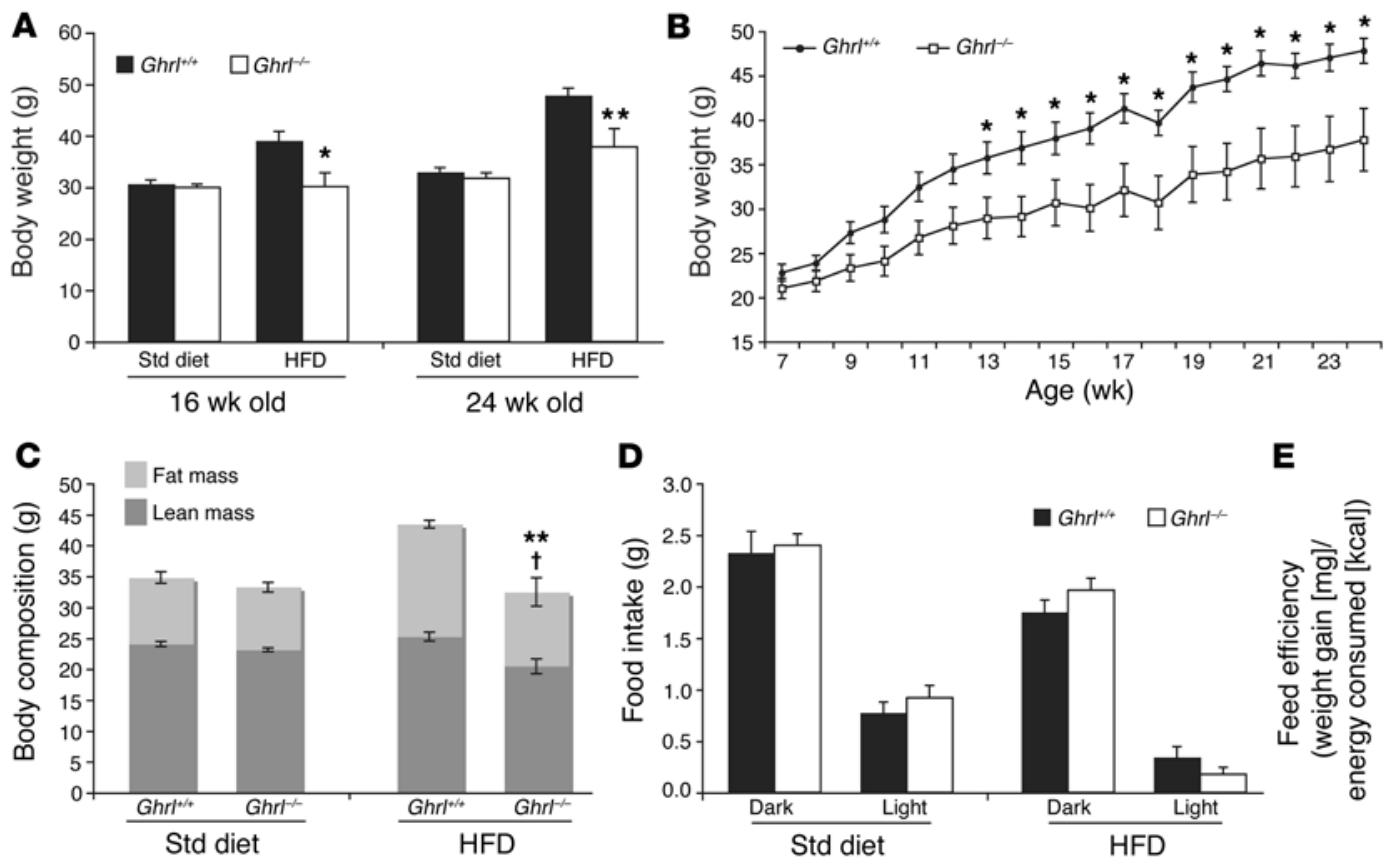

E

Figure 1

Differential effects of diet on body weight and body composition in male $\mathrm{Ghrl}^{-/-}$mice. (A) Body weights of male $\mathrm{Ghrl}^{+/+}$and $\mathrm{Ghrl}^{-/-}$mice maintained on standard (Std) diet were similar at 16 and 24 weeks of age ( $n=8 /$ genotype). When maintained on the HFD from 3 weeks after weaning, male $\mathrm{Ghrl}^{-/-}$mice showed a significant reduction in body weight compared with $\mathrm{Ghrl+/+}$ mice at 16 and 24 weeks of age $\left({ }^{\star \star} P<0.05 ; n=8 /\right.$ genotype). (B) Male $\mathrm{Ghrl}^{-1-}$ mice fed the HFD 3 weeks after weaning showed a significant reduction in body weight from 13 weeks of age onward; repeated measures ANOVA, effect of age $\times$ genotype: $F_{(1,17)}=5.895 ; P<0.0001\left({ }^{\star} P<0.05\right.$, post-hoc test). (C) Body composition of male $G h r l^{+/+}$and $\mathrm{Ghrl}^{-/-}$mice at 20 weeks of age on the standard diet was similar $(n=8 /$ genotype), whereas on the HFD, lean and fat mass were reduced in Ghrl-l- mice ( ${ }^{* *} P<0.05$ for fat mass; ${ }^{\dagger} P<0.01$ for lean mass; $n=8 /$ genotype). (D) Absolute food intake of male Ghrl+/+ and Ghrl-/- mice on the standard diet ( $n=4-5 /$ genotype) and HFD ( $n=8 /$ genotype) were similar during the light and dark periods. (E) Feed efficiency (calculated from caloric intake and body weight gain between 15 and 16 weeks of age) of $\mathrm{Ghrl}^{+/+}$and $\mathrm{Ghrl}^{-/-}$mice on the HFD $(n=7 / \mathrm{genotype})$.

on the HFD, absolute lean and fat mass were lower in the $\mathrm{Ghrl}^{-/-}$ mice compared with the $\mathrm{Ghrl}^{+/+}$mice, but when these measures were expressed as a percentage of total body weight, these changes reflected a specific decrease in percent adiposity, with no difference in percent lean mass between the 2 genotypes on this diet (lean mass: $\mathrm{Ghrl}^{+/+}, 54.3 \% \pm 0.9 \%$ vs. $\mathrm{Ghrl}^{-/-}, 59.2 \% \pm 2.4 \%$, NS; fat mass: $\mathrm{Ghrl}^{+/+}, 39.4 \% \pm 0.9 \%$ vs. Ghrl $\left.{ }^{-/-}, 30.9 \% \pm 3.5 \%, P<0.05\right)$. Despite the robust difference in body composition noted above, no differences were apparent in body length $(10.1 \pm 0.08 \mathrm{~cm}$ vs. $9.9 \pm 0.15 \mathrm{~cm}$; NS) or absolute 24-hour food intake (Figure 1D) between male $\mathrm{Ghrl}^{+++}$ and $\mathrm{Ghrl}^{-/-}$mice on the HFD. However, male $\mathrm{Ghrl}^{-/-}$mice on the HFD showed a trend $(P=0.06)$ toward decreased feed efficiency (defined as weight gain per kilocalorie consumed) compared with $\mathrm{Ghrl}^{+/+}$mice (Figure 1E).

Higher energy expenditure and activity in male Ghrl-1- mice on the $H F D$. Eighteen-week-old male Ghrl-/- mice maintained on the standard diet showed no differences in respiratory quotient (RQ), energy expenditure, or home cage activity (Figure 2, A, C, and E), compared with $\mathrm{Ghrl}^{+/+}$mice. When assessed after 3 months on the $\mathrm{HFD}, \mathrm{Ghrl}^{+/+}$and $\mathrm{Ghrl}^{-/-}$mice exhibited an equivalent reduction in RQ (Figure 2B). Despite the comparable level of lipid utilization, $\mathrm{Ghrl}^{-/-}$mice showed greater energy expenditure than $\mathrm{Ghrl}^{+/+}$mice during both the dark and light periods (Figure 2D). This difference in energy expenditure was also apparent when energy expenditure was expressed on a per mouse basis (dark period: $\mathrm{Gbrl}^{+/+}$, $1.186 \pm 0.047 \mathrm{kcal} / \mathrm{h}$ vs. $G \mathrm{hrl}^{-/-}, 1.307 \pm 0.030 \mathrm{kcal} / \mathrm{h}, P=0.0446$; light period, $\mathrm{Ghrl}^{+/+}, 0.973 \pm 0.024 \mathrm{kcal} / \mathrm{h}$ vs. $\mathrm{Ghrl}^{-/-} 1.118 \pm 0.048$ $\mathrm{kcal} / \mathrm{h}, P=0.0390)$. Part of this increased energy expenditure may be attributable to a greater level of locomotor activity compared with $\mathrm{Ghrl}^{+/+}$mice on this diet, which was apparent specifically during the dark (active) period (Figure 2F).

Male $\mathrm{Ghrl}^{-/-}$mice on a HFD exhibit an attenuated reduction in $\mathrm{GH}$ $m R N A$. We analyzed the expression of pituitary transcripts for $\mathrm{GH}$ and liver transcripts for GH receptor (GHR) and IGF-1 in male mice at 6 months of age. On the standard diet, no differences in $\mathrm{GH}$, IGF-1, or GHR mRNA were apparent between $\mathrm{Ghrl}^{+/+}$and $\mathrm{Ghrl}^{-/-}$ mice (Figure 3). Ghrl+/+ mice on the HFD demonstrated a marked reduction in GH and GHR expression compared with $\mathrm{Ghrl}^{+/+}$on a standard diet. However, Ghrl-1- mice on the HFD exhibited an attenuated reduction in GH mRNA, no reduction in GHR mRNA, and a trend toward increased IGF-1 mRNA levels (Figure 3).

Improved serum profile in male Ghrl-1- mice on a HFD. We investigated serum parameters in male mice at 24 weeks of age. $\mathrm{Ghrl}^{+/+}$ and $\mathrm{Ghrl}^{-/-}$mice maintained on standard chow showed no differences in various serum parameters, including glucose, insulin, and lipids (Table 1). These mice also showed similar glucose tolerance and insulin sensitivity (data not shown). In addition to the their increase in body weight and adiposity, $\mathrm{Ghrl}^{+/+}$mice fed the HFD demonstrated the expected increase in leptin, insulin, glucose, cholesterol, and alanine aminotransferase/aspartate aminotransferase (ALT/AST) ratio. Serum levels of IGF-1 were not different between the genotypes on either diet (Table 1). Despite their lower 
Std diet

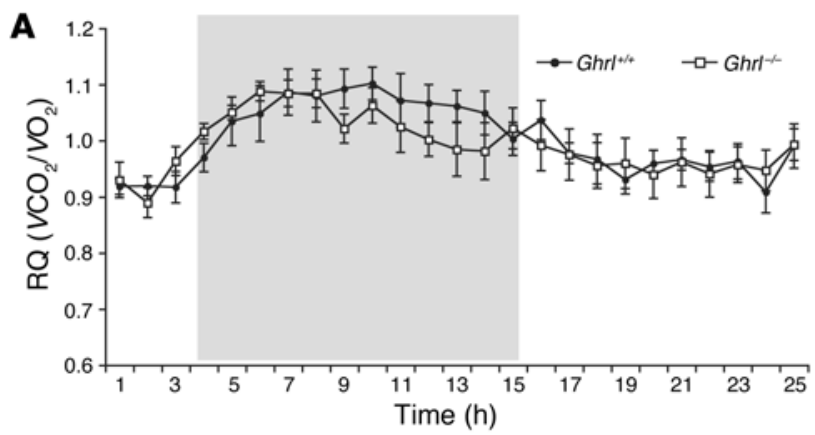

\section{C}

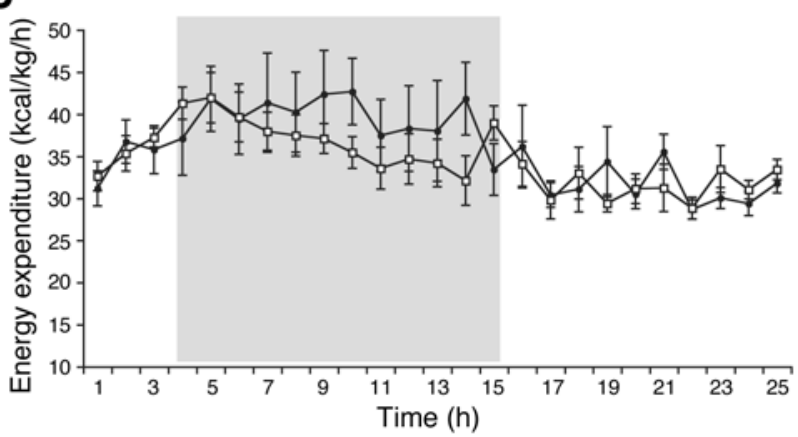

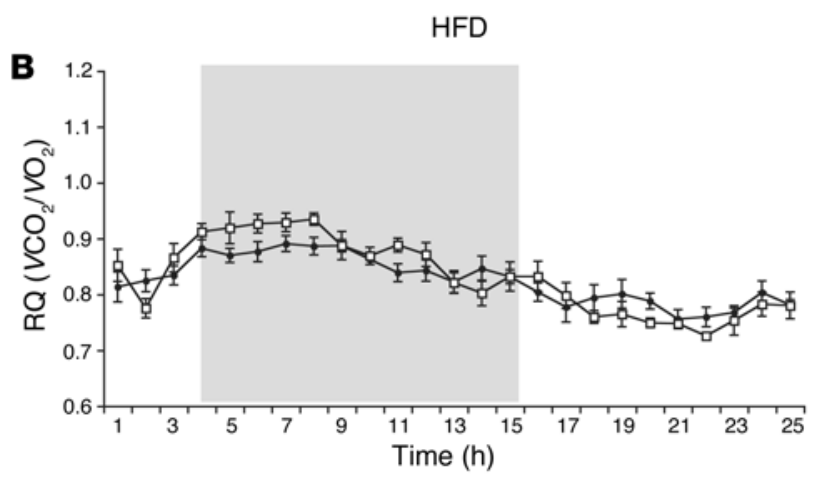

D

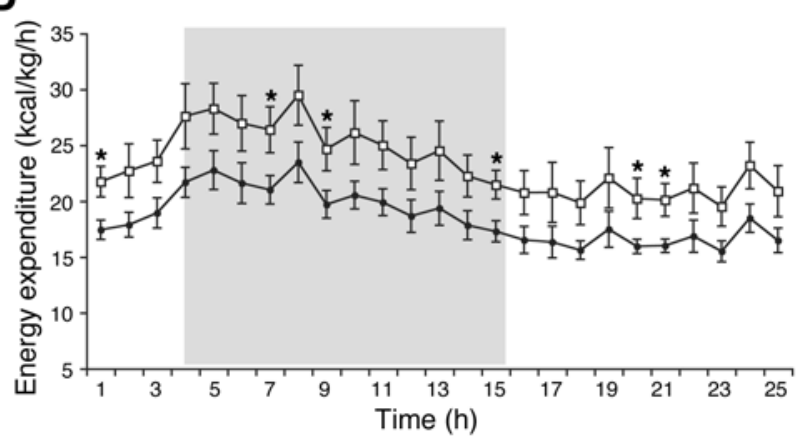

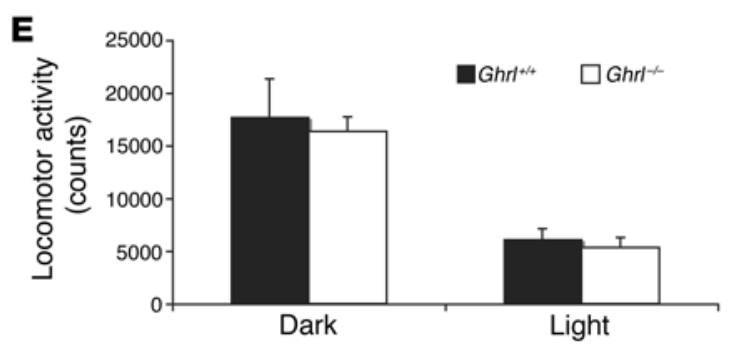

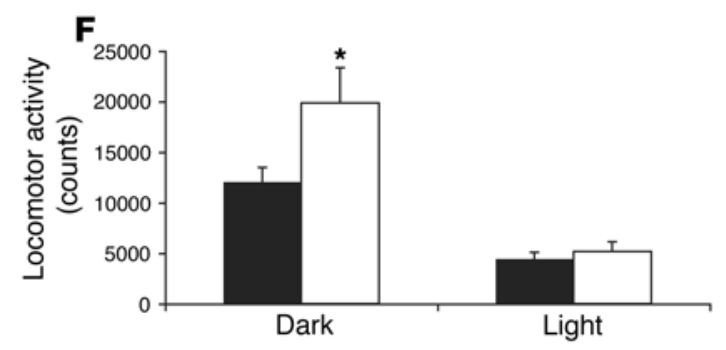

Figure 2

Metabolic parameters in 18-week-old male $\mathrm{Ghrl}^{-/-}$mice fed the standard diet or the HFD 3 weeks after weaning. RQs in male Ghrl+/+ and $\mathrm{Ghrl}^{-/-}$mice on the standard diet (A) and HFD (B) were similar. Energy expenditure in male Ghrl+/+ and Ghrl-/- mice on the standard diet (C) was similar, whereas on the HFD (D), energy expenditure was increased in Ghrl-- mice; repeated measures ANOVA, effect of genotype: $\mathrm{F}_{(1,14)}=5.062 ; P<0.05\left({ }^{*} P<0.05\right.$, post-hoc test). Locomotor activity was similar in male Ghrl+/+ and Ghrl/- mice on the standard diet (E), whereas activity was increased in male $\mathrm{Ghrl}^{-1-}$ mice fed the HFD (F); $P<0.05$. Gray bars represent the dark period. $n=5-7 /$ genotype for standard diet; $n=8$ /genotype for HFD.

accrual of body fat during exposure to the HFD, $\mathrm{Ghrl}^{-1-}$ mice also exhibited elevated levels of leptin, glucose, and cholesterol on the HFD, although these measures were significantly lower than those of $\mathrm{Ghrl}^{+/+}$mice on this diet (Table 1). Insulin and triglyceride levels were also significantly lower in $\mathrm{Ghrl}^{-/-}$mice compared with $\mathrm{Ghrl}^{+/+}$ mice on the HFD and, interestingly, also appeared lower compared with those of $\mathrm{Ghrl}^{-/-}$mice on the standard diet. These reduced insulin and triglyceride levels in the $\mathrm{Ghrl}^{-/-}$mice are consistent with the lower ALT/AST ratio of these mice on the HFD, which is a reflection of liver function and can be elevated in conditions of obesity (Table 1). $\mathrm{Ghrl}^{-/-}$mice on the HFD also exhibited improved glucose tolerance compared with $\mathrm{Ghrl}^{+/+}$mice (data not shown).

\section{Discussion}

The discrepant findings between the physiological and genetic studies of ghrelin on food intake and body weight have raised questions about the value of ghrelin as a potential therapeutic tar- get for obesity. Whereas ghrelin appears to have little impact on energy balance in mice exposed to a HFD as adults $(10,12)$, the present findings indicate that endogenous ghrelin plays a crucial role in regulating energy balance in male mice in response to early exposure at 6 weeks of age to a HFD.

We show that male $\mathrm{Ghrl}^{-/-}$mice switched to a HFD 3 weeks after weaning maintain a lean phenotype in adulthood. This finding extends our earlier data (12), which indicated a trend toward reduced body weight gain in older adult (18 weeks) $\mathrm{Ghrl}^{-/-}$mice placed on a HFD. The implications are that early exposure to a HFD is crucial to unmasking the resistance of $\mathrm{Ghrl}^{-/-}$mice to diet-induced obesity. In contrast to male mice, female $\mathrm{Ghrl}^{-/-}$mice placed on a HFD showed no difference in body composition compared with their wild-type counterparts. However, this finding could be due to the fact that these mice have a predominantly C57BL6/J (approximately 87.5\%) background. In contrast to male C57BL6/J mice, female mice of this strain gain very little weight on 
A
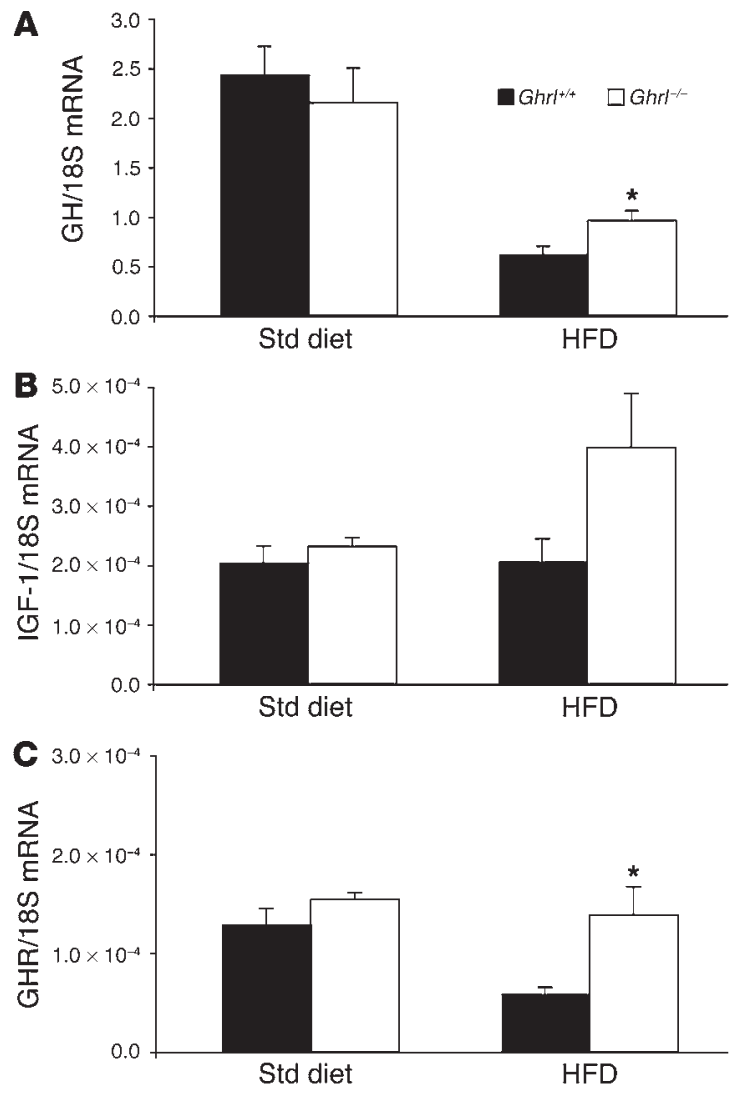

a HFD, making it inherently difficult to detect a lean phenotype in female mice in this background strain.

Consistent with their reduced body weight gain and adiposity, male $\mathrm{Ghrl}^{-1-}$ mice exhibited a trend for reduced feed efficiency. Although this finding was not statistically significant $(P=0.06)$, small differences in energy intake could contribute to the lean phenotype of these mice over time. Indeed, analyses of $\mathrm{Ghsr}^{-/}$mice weaned onto a HFD do show a significant reduction in feed efficiency over time in association with reduced body weight and adiposity (13). We also investigated possible changes in metabolic parameters. We have shown previously that exposure of adult $\mathrm{Ghrl}^{-/-}$mice to a HFD has a dramatic effect, lowering RQ after only 6 weeks on the diet (12), indicating that these mice are able to adapt to lipid substrate utilization more rapidly than their wild-type counterparts. However, in the present study, $\mathrm{Ghrl}^{+/+}$and $\mathrm{Ghrl}^{-/-}$ mice exhibited an equivalent respiratory quotient after 3 months on the HFD. Nevertheless, Ghrl-1- mice exhibited greater energy expenditure and locomotor activity than $\mathrm{Ghrl}^{+/+}$mice on the HFD. The increase in locomotor activity in the $\mathrm{Ghrl}^{-/-}$mice is consistent with exogenous ghrelin's reported suppression of spontaneous locomotor activity (14). This phenomenon likely contributes to the increase in energy expenditure during the dark (active) period. However, the $\mathrm{Ghrl}^{-/-}$mice also showed increased energy expenditure

\section{Figure 3}

The GH/IGF-1 axis in 6-month-old male $\mathrm{Ghrl}^{-/-}$mice fed a standard diet or HFD 3 weeks after weaning. (A) Pituitary GH mRNA in Ghrl+/+ and $\mathrm{Ghrl}^{-1-}$ mice on standard diet was similar, whereas on the HFD, $\mathrm{GH}$ mRNA was increased in $\mathrm{Ghrl}^{-/-}$compared with $\mathrm{Ghrl}^{+/+}$mice $\left.{ }^{\star} P<0.05\right)$. (B) IGF-1 mRNA in liver was similar in $\mathrm{Ghrl}^{+/+}$and $\mathrm{Ghrl}^{-/-}$ mice on the standard diet, whereas it showed a tendency to be increased in $\mathrm{Ghrl}^{-/-}$mice compared with $\mathrm{Ghrl}^{+/+}$mice fed a HFD. (C) GHR mRNA in the liver of $\mathrm{Ghrl}^{+/+}$and $\mathrm{Ghrl}^{-/-}$mice on standard diet was similar, whereas on the HFD, GHR mRNA was increased in $\mathrm{Ghrl}^{-1-}$ mice compared with $G h r l^{+/+}$mice $\left({ }^{*} P<0.05\right) . n=5-9 /$ genotype.

during the resting period when no differences in activity are apparent, suggesting that these mice inappropriately expend calories during this period. Studies have revealed that Ghsr-/- mice weaned onto a HFD also exhibit reduced energetic efficiency (13). Together these findings suggest that the ghrelin/GHSR pathway plays an important role in enabling mice to adapt to changes in nutritional status by controlling metabolic parameters such as energy expenditure and locomotor activity.

Ghrelin stimulates the release of GH (1). However, the importance of endogenous ghrelin in regulating $\mathrm{GH}$ in vivo remains unclear. GH also has well-documented effects on body composition and energy expenditure (15). Thus, the differential response of ghrelin-deficient animals to a HFD may, in fact, be due to perturbation in the GH/IGF-1 axis. Whereas mice of both genotypes exhibited a downregulation of GH and GHR mRNAs on the HFD, $\mathrm{GH}$ levels in $\mathrm{Ghrl}^{-/-}$mice were significantly higher than those of their wild-type counterparts. Considering that ghrelin is defined as a GH-releasing peptide (1), these findings are somewhat surprising. However, they are reminiscent of changes in the GH/IGF-1 axis observed in human obesity, which are commonly characterized by blunted basal GH secretion $(16,17)$. That GH expression in $\mathrm{Ghrl}^{-/-}$ mice is increased in association with reduced adiposity (relative to $\mathrm{Ghrl}^{+/+}$mice on HFD) is also consistent with the reduced adiposity observed in rats with GH infusion (18) or in transgenic mice overexpressing GHSR in GH-releasing hormone neurons, which results in GH overexpression (19). Considering that ghrelin levels are reduced by prolonged exposure to $\operatorname{HFD}(4,5)$, these collective data suggest that endogenous ghrelin may form part of a pathway involved in modulating the GH/IGF-1 pathway in response to changes in nutrient availability, such as those induced by a HFD. The lack of an equivalent reduction in GH and GHR in $\mathrm{Ghrl}^{-/-}$mice on a HFD may reflect the loss of signaling from ghrelin in this
Table 1

Serum parameters in male $\mathrm{Ghrl}^{+/+}$and $\mathrm{Ghrl}^{-/-}$mice following a 4-hour fast

$\begin{array}{lcccc} & \text { Ghrl++ Std diet } & \text { Ghrl-/-Std diet } & \text { Ghrl+/+ HFD } & \text { Ghrl-/ HFD } \\ \text { Leptin }(\mathrm{ng} / \mathrm{ml}) & 0.86 \pm 0.30 & 1.41 \pm 0.43 & 13.5 \pm 1.6 & 5.8 \pm 1.4^{\mathrm{A}} \\ \text { Insulin }(\mathrm{ng} / \mathrm{ml}) & 2.13 \pm 0.47 & 2.01 \pm 0.31 & 7.02 \pm 1.31 & 1.52 \pm 0.46^{\mathrm{A}} \\ \text { Glucagon }(\mathrm{ng} / \mathrm{ml}) & 0.015 \pm 0.0098 & 0.021 \pm 0.005 & 0.0143 \pm 0.0032 & 0.0104 \pm 0.0031 \\ \text { Glucose }(\mathrm{mg} / \mathrm{dl}) & 232 \pm 7 & 219 \pm 9 & 338 \pm 16 & 262 \pm 22^{\mathrm{B}} \\ \text { Triglycerides }(\mathrm{mg} / \mathrm{dl}) & 92 \pm 15 & 101 \pm 6 & 73 \pm 5 & 57 \pm 4^{\mathrm{B}} \\ \text { Cholesterol }(\mathrm{mg} / \mathrm{dl}) & 96 \pm 14 & 96 \pm 3 & 233 \pm 8 & 155 \pm 17^{\mathrm{A}} \\ \text { NEFAs }(\mathrm{mEg} / \mathrm{l}) & 0.62 \pm 0.11 & 0.76 \pm 0.05 & 0.57 \pm 0.02 & 0.58 \pm 0.02 \\ \text { ALT/AST } & 0.142 \pm 0.024 & 0.183 \pm 0.031 & 0.566 \pm 0.082 & 0.265 \pm 0.070^{\mathrm{B}} \\ \text { Total IGF-1 }(\mathrm{ng} / \mathrm{ml}) & 532 \pm 64 & 510 \pm 48 & 523 \pm 25 & 454 \pm 31\end{array}$

${ }^{A} P<0.001$ vs. $G h r l^{+++}$. ${ }^{B} P<0.05$ vs. Ghrl+++. NEFAs, nonesterified free fatty acids. 
pathway. Nevertheless, the finding that neither IGF-1 mRNA nor serum IGF-1 levels are significantly changed suggests that the perturbation in the GH/IGF-1 axis is most likely not responsible for the markedly lean phenotype of these mice.

In addition to decreased adiposity, conditions of elevated GH, such as acromegaly and GH overexpression in transgenic mice, are often accompanied by other abnormalities, such as insulin resistance and dyslipidemia (20). Thus, the higher expression of GH and GHR in the ghrelin-deficient mice on a HFD compared with their wild-type counterparts could lead to similar physiological changes. In contrast to these predictions, the $\mathrm{Ghrl}^{-{ }^{-}}$mice on the HFD showed improved levels of insulin, glucose, and lipids compared with wild-type mice on this diet and exhibited greater glucose tolerance. Therefore, $\mathrm{Ghrl}^{-/-}$mice showed no signs of insulin resistance or dyslipidemia, and we conclude that the changes in serum parameters are unlikely to be related to changes in $\mathrm{GH}$ expression and are more likely to be a direct result of the decreased adiposity of these mice. Thus, as opposed to a condition of elevated $\mathrm{GH}$, the $\mathrm{Ghrl}^{-1-}$ mice on a HFD more accurately reflect the maintenance of the GH/IGF-1 axis activity.

Hypothalamic neuropeptide systems that control food intake and metabolism undergo dynamic changes during the postnatal period $(21,22)$. Given that ghrelin has well-established effects on NPY/AgRP neurons in the hypothalamus $(8,9,23)$, it is plausible that the absence of ghrelin, like leptin (24), can impact on the functional organization of these pathways during development and alter the sensitivity of these pathways to subsequent changes in nutritional status. We would postulate that the effect of ghrelin on these metabolic pathways during development is likely to be mediated via the hypothalamic GHSR, since hypothalamus-specific Ghsr-deficient mice show a similar lean phenotype following early exposure to a HFD (13). Thus, our data, together with those of Zigman et al. (13), suggests that the absence of ghrelin signaling provides protection against diet-induced obesity induced by early exposure to a HFD, possibly due to dynamic changes in hypothalamic neuropeptide signaling $(21,22)$. It is not clear, however, why male $\mathrm{Ghrl}^{-/-}$mice exposed to a HFD 3 weeks after weaning maintain a lean phenotype, whereas older adult $\mathrm{Ghrl}^{-/}$mice remain susceptible to diet-induced obesity $(10,12)$. Nevertheless, it is well established that early-life nutrition has a profound impact on body weight, metabolism, long-term adiposity $(25,26)$, and even, longevity in mice (27).

In summary, we propose that ghrelin is a hormone that functions to protect against the debilitating effects of undernutrition by stimulating appetite and minimizing activity to conserve energy stores. In Western societies, humans typically consume diets very rich in fat and in overall caloric content. In this setting, the inability of the body to totally inhibit ghrelin's actions may not constitute a beneficial adaptive mechanism. Rather, ghrelin may contribute to a further increase in positive energy balance. Whereas the complete suppression of ghrelin would yield little evolutionary benefit for animals, the pharmacological inhibition of ghrelin action in humans provides a new avenue for antiobesity research.

\section{Methods}

Animals. Ghrl-/- mice were generated using the previously described highthroughput homologous recombination technology termed Velocigene (12, 28). After germ line transmission was established, mice were backcrossed to C57BL6/J mice to generate $\mathrm{N}_{3}$ breeding heterozygote mice that were set up in triads of 1 male and 2 females to generate homozygous null mice. All experiments reported were conducted on such $\mathrm{N}_{3} \mathrm{~F}_{2}$ littermates, which were housed under 12 hours of light per day in a temperature-controlled environment. The average litter size was 7.3 pups per triad, and mice were weaned at 21 days of age. All procedures were approved by the Regeneron Institutional Animal Care and Use Committee. Animals had free access to either standard chow (5020; Purina) or HFD (45\% fat, 4.7 kcal/g; 93075, Harlan Teklad).

Indirect calorimetry, food intake, and body composition. Metabolic parameters were obtained using an Oxymax (Columbus Instruments) open-circuit indirect calorimetry system as previously described (29). Briefly, $\mathrm{O}_{2}$ consumed $(\mathrm{ml} / \mathrm{kg} / \mathrm{h})$ and $\mathrm{CO}_{2}$ generated $(\mathrm{ml} / \mathrm{kg} / \mathrm{h})$ by each animal were measured for a 48 -hour period, and metabolic rate $\left(\mathrm{VO}_{2}\right)$ and RQ (ratio of $V \mathrm{CO}_{2}$ to $\mathrm{VO}_{2}$ ) were then calculated. Activity (counts) was also measured during the 48-hour period. Energy expenditure (or heat) was calculated as the product of the calorific value of oxygen $(=3.815+1.232 \times \mathrm{RQ})$ and the volume of $\mathrm{O}_{2}$ consumed. The first 24 -hour period allowed the mice to acclimate to the cages, and the data shown represent the second 24-hour period in the cages. Food intake was also assessed by automated measurements in metabolic cages, and body composition was determined in each individual animal utilizing dual-emission $\mathrm{x}$-ray absorption (PIXImus; Lunar). The calculation of feed efficiency in mice fed the HFD was determined by dividing body weight gain by caloric intake over a 7-day period.

Tissue and serum analysis. Serum samples were obtained from trunk blood taken following between 1300 and 1400 after a 4-hour fast and analyzed for glucose, triglycerides, cholesterol, ALT, and AST utilizing the Bayer 1650 blood chemistry analyzer. Nonesterified free fatty acids were analyzed by a diagnostic kit (Wako Pure Chemical Industries Ltd.) and insulin, leptin, and glucagon levels by LincoPlex (Linco Research Inc.). Serum total IGF-1 was measured using a diagnostic EIA kit (Diagnostic Systems Laboratories Inc.). GH, IGF-1, and GHR mRNA levels were measured by quantitative real-time RT-PCR as previously described $(30,31)$.

Data and statistics. Data are expressed as mean \pm SEM. Comparison of means was carried out using 2-tailed Student's $t$ test or ANOVA where appropriate, using the program StatView (Abacus Concepts). When a significant $\mathrm{F}$ ratio was obtained (significance $P<0.05$ ), post-hoc analysis was conducted between groups using a multiple comparison procedure with Bonferroni/Dunn posthoc comparison. $P$ values less than 0.05 were considered significant.

\section{Acknowledgments}

We thank Melissa Meola for the coordinated breeding of the ghrelin-knockout mice and Brian Ephraim for assistance with graphics.

Received for publication June 20, 2005, and accepted in revised form September 20, 2005.

Address correspondence to: Mark Sleeman, 777 Old Saw Mill River Road, Tarrytown, New York 10591, USA. Phone: (914) 345-7446; Fax: (914) 347-5045; E-mail: mark.sleeman@regeneron.com.

\footnotetext{
1. Kojima, M., et al. 1999. Ghrelin is a growth-hormone-releasing acylated peptide from stomach. Nature. 402:656-660.

2. Tschöp, M., Smiley, D.L., and Heiman, M.L. 2002. Ghrelin induces adiposity in rodents. Nature. 407:908-913.

3. Nakazato, M., et al. 2001. A role for ghrelin in the central regulation of feeding. Nature. 409:194-198.
}

4. Moesgaard, S.G., et al. 2004. Effects of high-fat feeding and fasting on ghrelin expression in the mouse stomach. Regul. Pept. 120:261-267.

5. Lee, H.M., Wang, G., Englander, E.W., Kojima, M., Greeley, G.H., Jr. 2002. Ghrelin, a new gastrointestinal endocrine peptide that stimulates insulin secretion: enteric distribution, ontogeny, influence of endocrine, and dietary manipulations. Endocri- nology. 143:185-190.

6. Willesen, M.G., Kristensen, P., and Romer, J. 1999. Co-localization of growth hormone secretagogue receptor and NPY mRNA in the arcuate nucleus of the rat. Neuroendocrinology.70:306-316.

7. Cone, R.D., et al. 2001. The arcuate nucleus as a conduit for diverse signals relevant to energy homeostasis. Int. J. Obes. Relat. Metab. Disord. 
25(Suppl. 5):S63-S67.

8. Cowley, M.A., et al. 2003. The distribution and mechanism of action of ghrelin in the CNS demonstrates a novel hypothalamic circuit regulating energy homeostasis. Neuron. 37:649-661.

9. Seoane, L.M., et al. 2003. Agouti-related peptide, neuropeptide $\mathrm{Y}$, and somatostatin-producing neurons are targets for ghrelin actions in the rat hypothalamus. Endocrinology. 144:544-551.

10. Sun, Y., Ahmed, S., and Smith, R.G. 2003. Deletion of ghrelin impairs neither growth nor appetite. Mol. Cell. Biol. 23:7973-7981.

11. Sun, Y., Wang, P., Zheng, H., and Smith, R.G. 2004 Ghrelin stimulation of growth hormone release and appetite is mediated through the growth hormone secretagogue receptor. Proc. Natl. Acad. Sci. U. S. A. 101:4679-4684.

12. Wortley, K.E., et al. 2004. Genetic deletion of ghrelin does not decrease food intake but influences metabolic fuel preference. Proc. Natl. Acad. Sci. U. S. A. 101:8227-8232.

13. Zigman, J.M., et al. 2005. Mice lacking ghrelin receptors resist the development of diet-induced obesity. J. Clin. Invest. 115:3564-3572. doi:10.1172/ JCI26002.

14. Tang-Christensen, M., et al. 2004. Central administration of ghrelin and agouti-related protein (83-132) increases food intake and decreases spontaneous locomotor activity in rats. Endocrinology. 145:4645-4652.

15. Mukherjee, A., Murray, R.D., and Shalet, S.M. 2004. Impact of growth hormone status on body composition and the skeleton. Horm. Res. 62(Suppl. 3):35-41

16. Scacchi, M., Pincelli, A.I., and Cavagnini, F. 1999. Growth hormone in obesity. Int. J. Obes. Relat. Metab. Disord. 23:260-271.

17. Veldhuis, J.D., et al. 1991. Dual defects in pulsatile growth hormone secretion and clearance subserve the hyposomatotropism of obesity in man. J. Clin. Endocrinol. Metab. 72:51-59.

18. Clark, R.G., et al. 1996. The obese growth hormone (GH)-deficient dwarf rat: body fat responses to patterned delivery of GH and insulin-like growth factor-I. Endocrinology. 137:1904-1912.

19. Lall, S., et al. 2004. Physiological studies of transgenic mice overexpressing growth hormone $(\mathrm{GH})$ secretagogue receptor $1 \mathrm{~A}$ in $\mathrm{GH}$-releasing hormone neurons. Endocrinology. 145:1602-1611.

20. Olsson, B., et al. 2005. Bovine growth hormone transgenic mice are resistant to diet-induced obesity but develop hyperphagia, dyslipidemia, and diabetes on a high-fat diet. Endocrinology. 146:920-930.

21. Grove, K.L., Allen, S., Grayson, B.E., and Smith, M.S. 2003. Postnatal development of the hypothalamic neuropeptide Y system. Neuroscience. 116:393-406.

22. Grove, K.L, and Smith, M.S. 2003. Ontogeny of the hypothalamic neuropeptide Y system. Physiol. Behav . 79:47-63.

23. Chen, H.Y., et al. 2004. Orexigenic action of peripheral ghrelin is mediated by neuropeptide $Y$ and agouti-related protein. Endocrinology. 145:2607-2612.

24. Pinto, S., et al. 2004. Rapid rewiring of arcuate nucle- us feeding circuits by leptin. Science. 304:110-115.

25. Ozanne, S.E., Lewis, R., Jennings, B.J., and Hales, C.N. 2004. Early programming of weight gain in mice prevents the induction of obesity by a highly palatable diet. Clin. Sci. (Lond.). 106:141-145.

26. Velkoska, E., Cole, T.J., and Morris, M.J. 2005. Early dietary intervention: long-term effects on blood pressure, brain neuropeptide $\mathrm{Y}$, and adiposity markers. Am. J. Physiol. Endocrinol. Metab. 288:E1236-E1243.

27. Ozanne, S.E., and Hales, C.N. 2004. Lifespan: catch-up growth and obesity in male mice. Nature. 427:411-412.

28. Valenzuela, D.M., et al. 2003. High-throughput engineering of the mouse genome coupled with high-resolution expression analysis. Nat. Biotechnol. 21:652-659.

29. Sleeman, M.W., et al. 2003. Ciliary neurotrophic factor improves diabetic parameters and hepatic steatosis and increases basal metabolic rate in $\mathrm{db} / \mathrm{db}$ mice. Proc. Natl. Acad. Sci. U. S. A. 100:14297-14302.

30. Iida, K., et al. 2004. Tissue-specific regulation of growth hormone $(\mathrm{GH})$ receptor and insulin-like growth factor-I gene expression in the pituitary and liver of GH-deficient (lit/lit) mice and transgenic mice that overexpress bovine $\mathrm{GH}(\mathrm{bGH})$ or a bGH antagonist. Endocrinology. 145:1564-1570.

31. Iida, K., Rosen, C.J., Ackert-Bicknell, C., and Thorner, M.O. 2005. Genetic differences in the insulinlike growth factor-I (IGF-I) gene among inbred strains of mice with different serum IGF-I levels. J. Endocrinol. 186:481-489. 